\title{
ADDENDUM
}

\section{Testing the accuracy of an observation-based classifier for rapid detection of autism risk}

M Duda, JA Kosmicki and DP Wall

Translational Psychiatry (2015) 5, e556; doi:10.1038/tp.2015.51; published online 28 April 2015

Addendum to: Translational Psychiatry (2014) 4: e424; doi:10.1038/tp.2014.65; published online 12 August 2014

In our recently published paper, ${ }^{1}$ we tested the performance of an observation-based classifier $(\mathrm{OBC})$ learned from machine-learning on archived answers to the Autism Diagnostic Observation Schedule (ADOS). Among other comparisons, we compared the OBC specificity and sensitivity to that reported by the Social Responsiveness Scale (SRS), a rapid instrument used in screening for autism risk. For this we used results published by Aldridge et al. $^{2}$ that demonstrated high sensitivity of the SRS, $>90 \%$, but a low specificity of $\sim 8 \%$ on a population of 48 children referred to a tertiary level. However, we missed a second paper $^{3}$ published in the same year that reported on a larger and more appropriate pediatric population. The Schanding et al. 2012 study found the sensitivity and specificity of SRS to be close to or $>90 \%$. These numbers are a more appropropriate reflection of the accuracy of the SRS for our comparisons and are similar to the reported accuracy of our OBC. Autism detection and diagnosis continues to need rapid, accurate and readily deployable tools. SRS and OBC each show potential to address the need individually or potentially in combination.

\section{REFERENCES}

1 Duda M, Kosmicki JA, Wall DP. Testing the accuracy of an observation-based classifier for rapid detection of autism risk. Transl Psychiatry 2014; 4: e424.

2 Aldridge FJ, Gibbs VM, Schmidhofer K, Williams M. Investigating the clinical usefulness of the Social Responsiveness Scale (SRS) in a tertiary level, autism spectrum disorder specific assessment clinic. J Autism Dev Disord 2012; 42, p 294-300.

3 Schanding GT Jr, Nowell KP, Goin-Kochel RP. Utility of the Social Communication Questionnaire-Current and Social Responsiveness Scale as Teacher-Report Screening Tools for Autism Spectrum Disorders. J Autism Dev Disord 2012; 42: 1705-1716. 